\title{
Risk Factors for Revisiting After Emergency Department Discharge: a Longitudinal Retrospective Chart Review
}

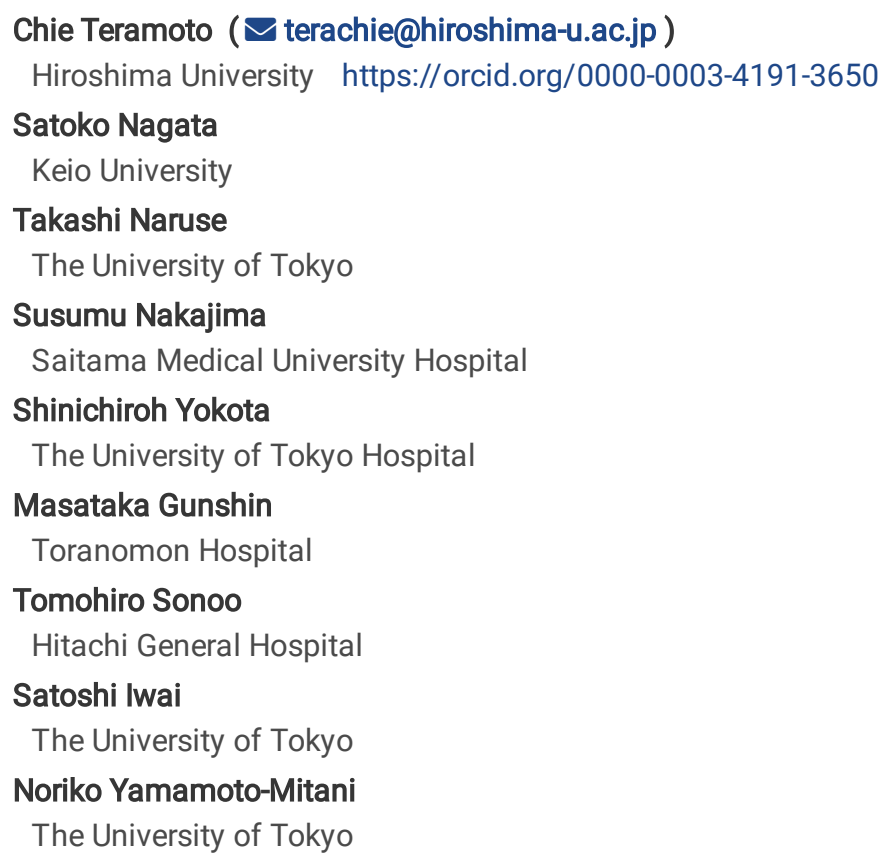

Research article

Keywords: emergency departments, access to care, primary care, patient support, revisits, patients discharge

Posted Date: August 25th, 2020

DOI: https://doi.org/10.21203/rs.3.rs-62406/v1

License: (c) (i) This work is licensed under a Creative Commons Attribution 4.0 International License. Read Full License 


\section{Abstract}

Background: Emergency department (ED) patients are often discharged after treatment for minor illnesses. However, many of these patients revisit the ED. This study aimed to describe ED revisit rate and investigate the risk factors for 3-day/30-day ED revisit.

Methods: A longitudinal retrospective chart review was used to extract data regarding predisposing factors (e.g. gender, age), enabling factors (e.g. economic status, ambulance use) and need factors (e.g. diagnosis at ED discharge, history of diseases), from the medical charts of patients discharged from an ED in Tokyo, Japan, from February to December 2013. Multiple logistic regression analyses were used to predict 3-day/30-day ED revisit based on initial visit information.

Results: During the studied period, 8,754 patients were discharged from the ED, of whom $48.8 \%$ were men, and the mean age was 39.7 years. Of these, 338 (3.9\%) revisited the ED within three days and 682 (7.8\%) revisited the ED within 30 days of discharge. In the multiple logistic regression analysis, risk factors for 3-day ED revisits were: having a history of same hospital use, arriving by ambulance, having a common problem (e.g. fever), and history of asthma. Risk factors for 30-day ED revisits were using health care services and having a history of same hospital use.

Conclusions: In this study, the rate of ED revisit was less than that reported by other studies, which might be because most patients included in this study usually visited the hospital. Our results show that the predictive factors for 3-day/30-day ED revisit in Japan were similar to those in other countries. Patients with the abovementioned factors should be carefully screened on their initial ED visit, and some follow-up care in clinical and community settings should be arranged.

\section{Background}

The increasing number of patients who visit the emergency department (ED) in recent years is a social problem worldwide. In Organization for Economic Cooperation and Development (OECD) countries, there has been an increase in the number of ED patients with low urgency problems, who tend to be discharged home [1]. In a similar trend, $80.6 \%$ of patients were discharged from secondary and tertiary ED in Japan [2], and owing to its aging population these numbers will increase in the future. Patients discharged from the ED were more likely to have reportedly lower health-related quality of life [3] and were in the high-risk group regarding death, hospitalization, and ED revisit after ED discharge [4]. Thus, it is important to consider the establishment of a follow-up system for patients discharged from ED.

A previous review of ED patients' revisit rate found that 3.1\%-13.1\% returned within three days, $4.4 \%$ within 28 days and $23.2 \%-32.5 \%$ within 90 days [4]. Particularly, the lower the revisit rate within the first three days post discharge is considered to be an indicator of higher quality emergency medical care [4]. Internationally, screening tools for ED revisit have been developed [5].

It is important to understand the characteristics of ED revisit patients in the Japanese medical system. Although previous studies in Japan have focused on ED revisits among special groups, i.e., psychiatry patients [6], and asthmatic patients [7], a wide variety of patients visit the ED, from the neonatal to older adults. A general population approach regarding ED revisits in Japan is necessary. Readmission within four weeks is considered similar to ED revisits and has been used to assess the value of quality services to inpatients in general [8]. A program of transitional care which comprises patient support for 30 days after discharge has been developed in other countries [9]. We postulated that by assessing the ED revisit rate within 30 days after going home, we could evaluate the necessity of the at-home support after being discharged.

\section{Objectives}

This study investigated the rate of ED revisits its related factors in Japan for the general population among patients discharged from a university hospital ED.

Previous studies reported that a 3-day ED revisit can be a quality indicator for the services ED physicians provided [4], and may not be related to the disease itself. Hence, we separated the three groups to focus on the "30-day ED revisit" group (ED revisit patients within 0-3 days, 4-30 days, or not within 30 days).

\section{Methods}

\section{Study design}

A longitudinal retrospective chart review was conducted with patient records obtained from January 1, 2013 to March 31, 2014. This article was reported based on The Reporting of studies Conducted using Observational Routinely-collected health Data (RECORD) Statement [10]. 
This study was conducted in a university hospital, in a critical care center ED in Tokyo, Japan. The hospital was a special function hospital with 1,163 general beds, which received 16,552 patients in 2018 [11].

\section{Participants}

Inclusion criteria were: 1) ED visit from February 1 to December 31, 2013; 2) no ED hospital visit during previous month; 3) discharged home or to another homecare setting (but not other hospitals) after the initial ED visit.

Recruitment occurred in the following five steps: 1) collecting patient ID (patient unit data) by ED visit system from January 1, 2013 to March 31,$2014 ; 2$ ) matching the data to Patient ID; 3 ) assigning an anonymized study ID to each patient; 4) identifying the initial ED visit date of each patient; 5) excluding patients whose initial ED visit was not in the selected study range.

\section{Outcome measurements}

ED revisit was measured in three categories: patients who revisited the study hospital ED within three days were set to the "A: 3-day" group, 430 days ED discharge patients were placed in the "B: 30-day" group, and patients not revisiting within 30 days formed the "C: no revisit" group.

\section{Variables \& Data cleaning methods}

We organized the items based on Andersen's [12] behavioral model of health services use: predisposing characteristics, demographic factors, social factors, health beliefs, enabling characteristics; financing, economic status, organization, need characteristics, perceived need and evaluated need, including the Japan Triage and Acuity Scale (JTAS) [13]. An additional table file shows complete list of codes and data cleaning methods in more detail [see Additional file 1].

\section{Data sources, Data access, \& Linkage}

We collected the medical chart information of ED patients through a "ED patient visit system" which included an ED visit date and patient ID. Using the patient ID, we retrieved 11 CSV files by the Department of Healthcare Information Management of the hospital. The data set included three databases: 1) the ED patient visit system: age, gender, ED visit information, diagnosis, outcome, among others; 2) the electronic medical record system describing data by ED doctors; 3 ) the ordering system, including medical examination, injection ordered and treatment. All data were linked through patient ID.

\section{Bias \& Study size}

There was no bias in this study's outcome "ED revisit," because of the complete data surveyed during study period. On the other hand, information quality could vary because of input differences from ED doctors.

\section{Statistical methods}

Descriptive statistics, frequency counts, percentage rates, means, standard deviation [SD], and range were calculated for each variable at the time of the initial ED visit and transition of ED revisits within 90 days after ED discharge. ED revisit cumulative incidence was described by frequency count until 90 days in those discharged from the ED after the initial visit. We compared the three groups of data at the time of initial ED visit by chi-square tests, and one-way ANOVAs. A multiple logistic regression analysis (backward elimination) was used for initial ED visit to examine the risk factor of group $\mathrm{A}$, and to examine the characteristics of $\mathrm{B}$, compared with $\mathrm{C}$. The $p$-value $<0.01$ was defined as significant, because of big sample size and many analyses. All data were analyzed using IBM SPSS Statistics 24 (IBM Corp., Armonk, NY, USA).

\section{Results}

\section{Participants}

The flow of participants included in this study is shown in Figure 1. There were 17,437 cases (13,180 patients) who visited the ED from February to December 2013.

Figure. 1 Flow of participants

Patients who were hospitalized, transferred to another hospital, or died were excluded. We also excluded patients whose initial ED visit occurred in January 2013, and finally, analyzed 11,322 cases (8,754 patients) who were discharged from the ED after an initial visit. Table 1 shows the characteristics of ED patients in this study. 


\begin{tabular}{|c|c|c|c|}
\hline Variables & \multirow[b]{2}{*}{ Male } & \multicolumn{2}{|c|}{$\begin{array}{l}\mathrm{n}(\%) \\
\text { mean (SD) } \\
\text { (range) }\end{array}$} \\
\hline Gender, n (\%) & & 4275 & $(48.8)$ \\
\hline Female & 4479 & $(51.2)$ & \\
\hline Age & 39.7 & $(26.0)$ & \\
\hline (Min-Max) & $\varangle 0-$ & 100) & \\
\hline Age group, n (\%) & Child (0-14 years) & 1810 & $(20.7)$ \\
\hline Adult (15-64 years) & 4930 & $(56.3)$ & \\
\hline Older Adult ( $\geq 65$ years) & 2014 & $(23.0)$ & \\
\hline \multirow[t]{3}{*}{ History of same hospital use, $n(\%)$} & No history & 3568 & $(40.8)$ \\
\hline & User of the hospital & 4932 & $(56.3)$ \\
\hline & Missing data & 254 & $(2.9)$ \\
\hline Date, n (\%) & Weekdays & 4911 & $(56.1)$ \\
\hline Weekend & 3843 & $(43.9)$ & \\
\hline Shift time, n (\%) & Night shift & 5359 & $(61.2)$ \\
\hline Day shift & 3395 & $(38.8)$ & \\
\hline Time, n (\%) & In time & 1483 & $(16.9)$ \\
\hline Out of time & 7271 & $(83.1)$ & \\
\hline \multirow[t]{2}{*}{ Method to the ED, n (\%) } & Walk-in & 5955 & $(68.0)$ \\
\hline & Ambulance & 2799 & $(32.0)$ \\
\hline JTAS, n (\%) & Resuscitation-Urgency & 3609 & $(41.2)$ \\
\hline Less urgent/Non-urgent & 4311 & $(49.2)$ & \\
\hline Missing data & 834 & (9.5) & \\
\hline
\end{tabular}

Note: $\mathrm{n}(\%)$ or mean (SD)

\section{ED revisits after discharge}

Figure 2 shows the transition of ED revisits within 90 days after discharge. Of the 8,754 ED discharge patients who revisited the ED, 53 (0.6\%) returned on the same day, $338(3.9 \%)$ within three days, $456(5.2 \%)$ within seven days, $544(6.2 \%)$ within 14 days, $682(7.8 \%)$ within 30 days, $881(10.1 \%)$ within 60 days, and $1,022(11.7 \%)$ within 90 days.

Figure. 2 Transition of emergency department revisits within 90 days after discharge

Among ED revisit patients, patients who revisited within three days may differ from those who revisited after four days, given how groups were disposed. Table 2 shows the results of comparison between the three groups. Group $\mathrm{C}$ had less women $(50.8 \%, p=0.04)$ than $\mathrm{A}$ or $\mathrm{B}$, whereas B had an older mean age of 47.2 years $(p<0.01)$ and more older adults $(34.6 \%, p<0.01)$ than A or C.

Table 2 Comparison between group A and B: data from initial visit and revisit $₫ n=8,754$ ) 


\begin{tabular}{|c|c|c|c|c|c|c|c|c|c|}
\hline \multirow[t]{2}{*}{$\begin{array}{l}\text { Andersen's } \\
\text { behavioral model }\end{array}$} & & & \multicolumn{2}{|c|}{$\begin{array}{l}\text { A. 3-day } \\
\text { ED revisit } \\
(n=338)\end{array}$} & \multicolumn{2}{|c|}{$\begin{array}{l}\text { B. 30-day } \\
\text { ED revisit } \\
(n=344)\end{array}$} & \multicolumn{2}{|c|}{$\begin{array}{l}\text { C. No revisit } \\
(n=8,072)\end{array}$} & \multirow[b]{2}{*}{$p$-value } \\
\hline & & & $\mathrm{n}$ & $(\%)$ & $\mathrm{n}$ & $(\%)$ & $\mathrm{n}$ & $(\%)$ & \\
\hline \multicolumn{10}{|l|}{ Predisposing } \\
\hline \multirow[t]{7}{*}{ Demographic } & Gender & Male & 152 & $(45.0)$ & 150 & $(43.6)$ & 3973 & $(49.2)$ & $0.04^{\mathrm{a}}$ \\
\hline & & Female & 186 & $(55.0)$ & 194 & $(56.4)$ & 4099 & $(50.8)$ & \\
\hline & Age & mean (SD) & 42.0 & $(12.4)$ & 47.2 & $(27.1)$ & 39.3 & $(25.9)$ & $<0.01^{b}$ \\
\hline & & (Min-Max) & $(0-$ & 94) & $(0-$ & 100) & $(0-$ & 99) & \\
\hline & Age group & Child (0-14 years) & 66 & $(19.5)$ & 49 & $(14.2)$ & 1695 & $(21.0)$ & $<0.01^{\mathrm{a}}$ \\
\hline & & $\begin{array}{l}\text { Adult (15-64 } \\
\text { years) }\end{array}$ & 187 & $(55.3)$ & 176 & $(51.2)$ & 4567 & $(56.6)$ & \\
\hline & & $\begin{array}{l}\text { Older Adult (over } \\
65 \text { years) }\end{array}$ & 85 & $(25.1)$ & 119 & $(34.6)$ & 1810 & $(22.4)$ & \\
\hline \multirow[t]{6}{*}{ Social } & $\begin{array}{l}\text { Marital } \\
\text { Status }\end{array}$ & No partner & 104 & $(30.8)$ & 95 & $(27.6)$ & 2108 & $(26.1)$ & $<0.01^{\mathrm{a}}$ \\
\hline & & With partner & 112 & $(33.1)$ & 149 & $(43.3)$ & 1639 & $(20.3)$ & \\
\hline & Job Status & No work/study & 101 & $(29.9)$ & 116 & $(33.7)$ & 1789 & $(22.2)$ & $<0.01^{\mathrm{a}}$ \\
\hline & & working/studying & 107 & $(31.7)$ & 114 & $(33.1)$ & 1983 & $(24.6)$ & \\
\hline & $\begin{array}{l}\text { Household } \\
\text { members }\end{array}$ & Living alone & 25 & $(7.4)$ & 34 & (9.9) & 310 & (3.8) & $<0.01^{\mathrm{a}}$ \\
\hline & & $\begin{array}{l}\text { Living with } \\
\text { family }\end{array}$ & 195 & $(57.7)$ & 218 & $(63.4)$ & 3524 & $(43.7)$ & \\
\hline \multirow[t]{2}{*}{ Beliefs } & $\begin{array}{l}\text { History of } \\
\text { same }\end{array}$ & No & 109 & $(32.2)$ & 60 & $(17.4)$ & 3859 & $(47.8)$ & $<0.01^{\mathrm{a}}$ \\
\hline & hospital use & Yes & 229 & $(67.8)$ & 284 & $(82.6)$ & 4213 & $(52.2)$ & \\
\hline \multicolumn{10}{|l|}{ Enabling } \\
\hline \multirow[t]{2}{*}{ Financing } & $\begin{array}{l}\text { Economic } \\
\text { status }\end{array}$ & No problem & 330 & $(97.6)$ & 100 & $(29.1)$ & 7993 & $(99.0)$ & $<0.01^{\mathrm{a}}$ \\
\hline & & Problematic & 8 & $(2.4)$ & 19 & (5.5) & 79 & $(1.0)$ & \\
\hline \multirow[t]{12}{*}{ Organization } & Date & Weekdays & 185 & $(54.7)$ & 207 & $(60.2)$ & 4519 & $(56.0)$ & $0.27^{a}$ \\
\hline & & Weekend & 153 & $(45.3)$ & 137 & $(39.8)$ & 3553 & $(44.0)$ & \\
\hline & Sift time & Night shift & 206 & $(60.9)$ & 189 & $(54.9)$ & 4964 & $(61.5)$ & $0.05^{a}$ \\
\hline & & Day shift & 132 & $(39.1)$ & 155 & $(45.1)$ & 3108 & $(38.5)$ & \\
\hline & Time & In time & 54 & $(16.0)$ & 63 & $(18.3)$ & 1366 & $(16.9)$ & $0.71^{\mathrm{a}}$ \\
\hline & & Out of time & 284 & $(84.0)$ & 281 & $(81.7)$ & 6706 & $(83.1)$ & \\
\hline & $\begin{array}{l}\text { Method to } \\
\text { ED visit }\end{array}$ & Walk-in & 219 & $(64.8)$ & 257 & $(74.7)$ & 5479 & $(67.9)$ & $0.01^{a}$ \\
\hline & & Ambulance & 119 & $(35.2)$ & 87 & $(25.3)$ & 2593 & $(32.1)$ & \\
\hline & Companies & Alone & 72 & $(21.3)$ & 72 & $(20.9)$ & 1742 & $(21.6)$ & $0.41^{\mathrm{a}}$ \\
\hline & & With someone & 159 & $(47.0)$ & 151 & $(43.9)$ & 3880 & $(48.1)$ & \\
\hline & $\begin{array}{l}\text { Using health } \\
\text { care services }\end{array}$ & No services & 306 & $(90.5)$ & 289 & $(84.0)$ & 7755 & $(96.1)$ & $<0.01^{\mathrm{a}}$ \\
\hline & & With services & 32 & (9.5) & 55 & $(16.0)$ & 317 & (3.9) & \\
\hline
\end{tabular}




\begin{tabular}{|c|c|c|c|c|c|c|c|c|c|}
\hline \multirow[t]{17}{*}{ Perceived } & Sign & Cardiovascular & 17 & $(5.0)$ & 29 & $(8.4)$ & 406 & (5.0) & $0.02^{\mathrm{a}}$ \\
\hline & & ENT-Ears & 4 & $(1.2)$ & 0 & $(0.0)$ & 93 & $(1.2)$ & $0.13^{\mathrm{a}}$ \\
\hline & & $\begin{array}{l}\text { ENT-Mouth, } \\
\text { throat, neck }\end{array}$ & 16 & $(4.7)$ & 14 & $(4.1)$ & 542 & (6.7) & $0.06^{\mathrm{a}}$ \\
\hline & & ENT-Nose & 4 & $(1.2)$ & 3 & $(0.9)$ & 137 & $(1.7)$ & $0.40^{\mathrm{a}}$ \\
\hline & & Environmental & 1 & $(0.3)$ & 1 & $(0.3)$ & 61 & $(0.8)$ & $0.39^{a}$ \\
\hline & & Gastrointestinal & 52 & $(15.4)$ & 46 & $(13.4)$ & 1183 & $(14.7)$ & $0.75^{\mathrm{a}}$ \\
\hline & & Genitourinary & 8 & $(2.4)$ & 15 & $(4.4)$ & 241 & $(3.0)$ & $0.27^{a}$ \\
\hline & & Mental Health & 2 & $(0.6)$ & 0 & $(0.0)$ & 31 & $(0.4)$ & $0.41^{\mathrm{a}}$ \\
\hline & & Neurologic & 47 & $(13.9)$ & 49 & $(14.2)$ & 1390 & $(17.2)$ & $0.30^{\mathrm{a}}$ \\
\hline & & OB-GYN & 18 & (5.3) & 23 & $(6.7)$ & 288 & (3.6) & $<0.01^{\mathrm{a}}$ \\
\hline & & Ophthalmologic & 10 & (3.0) & 10 & $(2.9)$ & 405 & (5.0) & $0.05^{a}$ \\
\hline & & Orthopedic & 20 & $(5.9)$ & 24 & $(7.0)$ & 748 & (9.3) & $0.04^{\mathrm{a}}$ \\
\hline & & Respiratory & 19 & (5.6) & 27 & $(7.8)$ & 423 & $(5.2)$ & $0.06^{\mathrm{a}}$ \\
\hline & & Skin & 24 & $(7.1)$ & 20 & $(5.8)$ & 727 & $(9.0)$ & $0.07^{a}$ \\
\hline & & $\begin{array}{l}\text { Substance } \\
\text { Misuse }\end{array}$ & 1 & $(0.3)$ & 1 & $(0.3)$ & 32 & $(0.4)$ & $0.04^{\mathrm{a}}$ \\
\hline & & Trauma & 10 & (3.0) & 5 & (1.5) & 212 & $(2.6)$ & $0.38^{a}$ \\
\hline & & $\begin{array}{l}\text { Common } \\
\text { Problem }\end{array}$ & 68 & $(20.1)$ & 63 & $(18.3)$ & 1013 & $(12.5)$ & $<0.01^{\mathrm{a}}$ \\
\hline \multirow[t]{14}{*}{ Evaluated } & \multirow{2}{*}{$\begin{array}{l}\text { Some } \\
\text { Departments } \\
\text { use }\end{array}$} & $\begin{array}{l}\text { One department } \\
\text { use }\end{array}$ & 311 & $(92.0)$ & 319 & $(92.7)$ & 7450 & (92.3) & $0.94^{\mathrm{a}}$ \\
\hline & & $\begin{array}{l}\text { Some } \\
\text { departments use }\end{array}$ & 27 & (8.0) & 25 & (7.3) & 622 & (7.7) & \\
\hline & \multirow[t]{5}{*}{ JTAS } & Resuscitation & 5 & (1.5) & 2 & $(0.6)$ & 98 & $(1.2)$ & $0.22^{\mathrm{a}}$ \\
\hline & & Emergency & 35 & $(10.4)$ & 23 & $(6.7)$ & 536 & $(6.6)$ & \\
\hline & & $\begin{array}{l}\text { Semi emergency } \\
\text { 『ref.) }\end{array}$ & 114 & $(33.7)$ & 110 & $(32.0)$ & 2686 & (33.3) & \\
\hline & & Low emergency & 122 & $(36.1)$ & 134 & $(39.0)$ & 3193 & $(39.6)$ & \\
\hline & & Non-emergency & 26 & $(7.7)$ & 35 & $(10.2)$ & 801 & $(9.9)$ & \\
\hline & \multirow[t]{7}{*}{ Diagnosis } & $\begin{array}{l}\text { I Infectious } \\
\text { diseases }\end{array}$ & 30 & (8.9) & 24 & (7.0) & 664 & (8.2) & $0.64^{\mathrm{a}}$ \\
\hline & & II Neoplasms & 7 & $(2.1)$ & 7 & $(2.0)$ & 105 & (1.3) & $0.27^{a}$ \\
\hline & & $\begin{array}{l}\text { III Diseases of } \\
\text { the blood }\end{array}$ & 2 & $(0.6)$ & 4 & $(1.2)$ & 21 & $(0.3)$ & $<0.01^{\mathrm{a}}$ \\
\hline & & $\begin{array}{l}\text { IV Metabolic } \\
\text { diseases }\end{array}$ & 4 & $(1.2)$ & 8 & (2.3) & 116 & $(1.4)$ & $0.37^{a}$ \\
\hline & & $\begin{array}{l}\text { V Mental } \\
\text { disorders }\end{array}$ & 8 & $(2.4)$ & 11 & (3.2) & 208 & $(2.6)$ & $0.75^{\mathrm{a}}$ \\
\hline & & $\begin{array}{l}\text { VI Nervous } \\
\text { system }\end{array}$ & 7 & $(2.1)$ & 4 & $(1.2)$ & 178 & $(2.2)$ & $0.43^{a}$ \\
\hline & & $\begin{array}{l}\text { VII Eye and } \\
\text { adnexa }\end{array}$ & 9 & $(2.7)$ & 8 & (2.3) & 280 & (3.5) & $0.39^{a}$ \\
\hline
\end{tabular}




\begin{tabular}{|c|c|c|c|c|c|c|c|c|}
\hline & VIII Ear & 6 & (1.8) & 3 & $(0.9)$ & 166 & (2.1) & $0.29^{a}$ \\
\hline & $\begin{array}{l}\text { IX Circulatory } \\
\text { system }\end{array}$ & 9 & $(2.7)$ & 23 & (6.7) & 274 & $(3.4)$ & $<0.01^{a}$ \\
\hline & $\begin{array}{l}\text { X Respiratory } \\
\text { system }\end{array}$ & 45 & (13.3) & 47 & (13.7) & 779 & (9.7) & $<0.01^{\mathrm{a}}$ \\
\hline & $\begin{array}{l}\text { XI Digestive } \\
\text { system }\end{array}$ & 28 & (8.3) & 28 & (8.1) & 469 & $(5.8)$ & $0.04^{\mathrm{a}}$ \\
\hline & XII Skin & 11 & (3.3) & 5 & $(1.5)$ & 354 & (4.4) & $0.02^{\mathrm{a}}$ \\
\hline & $\begin{array}{l}\text { XIII } \\
\text { Musculoskeletal }\end{array}$ & 10 & (3.0) & 15 & $(4.4)$ & 219 & (2.7) & $0.19^{\mathrm{a}}$ \\
\hline & $\begin{array}{l}\text { XIV Genitourinary } \\
\text { system }\end{array}$ & 23 & $(6.8)$ & 22 & $(6.4)$ & 493 & (6.1) & $0.86^{\mathrm{a}}$ \\
\hline & $\begin{array}{l}\text { XV Pregnancy, } \\
\text { childbirth }\end{array}$ & 12 & (3.6) & 22 & $(6.4)$ & 190 & (2.4) & $<0.01^{a}$ \\
\hline & $\begin{array}{l}\text { XVI Perinatal } \\
\text { period }\end{array}$ & 0 & $(0.0)$ & 1 & $(0.3)$ & 2 & $(0.0)$ & $0.03^{b}$ \\
\hline & XVII Congenital & 0 & $(0.0)$ & 2 & $(0.6)$ & 7 & $(0.1)$ & $0.02^{b}$ \\
\hline & $\begin{array}{l}\text { XVIII Symptoms, } \\
\text { signs }\end{array}$ & 70 & (20.1) & 47 & $(13.7)$ & 1163 & (14.4) & $<0.01^{\mathrm{a}}$ \\
\hline & $\begin{array}{l}\text { XIX Injury, } \\
\text { poisoning }\end{array}$ & 55 & (16.3) & 59 & $(17.2)$ & 2298 & (28.5) & $0.58^{\mathrm{a}}$ \\
\hline & $\begin{array}{l}\text { XXI Health } \\
\text { services }\end{array}$ & 2 & $(0.6)$ & 3 & $(0.9)$ & 62 & $(0.8)$ & $0.91^{\mathrm{a}}$ \\
\hline \multirow{16}{*}{$\begin{array}{l}\text { Disease } \\
\text { History }\end{array}$} & Dementia & 7 & $(2.1)$ & 16 & $(4.7)$ & 156 & (1.9) & $<0.01^{\mathrm{a}}$ \\
\hline & $\begin{array}{l}\text { Myocardial } \\
\text { infarction }\end{array}$ & 12 & (3.6) & 15 & $(4.4)$ & 158 & $(2.0)$ & $<0.01^{\mathrm{a}}$ \\
\hline & $\begin{array}{l}\text { Congestive heart } \\
\text { failure }\end{array}$ & 57 & (16.9) & 68 & $(19.8)$ & 724 & $(9.0)$ & $<0.01^{\mathrm{a}}$ \\
\hline & $\begin{array}{l}\text { Peripheral } \\
\text { vascular disease }\end{array}$ & 8 & (2.4) & 13 & (3.8) & 69 & $(0.9)$ & $<0.01^{\mathrm{a}}$ \\
\hline & $\begin{array}{l}\text { Cerebrovascular } \\
\text { disease }\end{array}$ & 30 & (8.9) & 41 & (11.9) & 428 & (5.3) & $<0.01^{\mathrm{a}}$ \\
\hline & $\begin{array}{l}\text { Chronic } \\
\text { pulmonary } \\
\text { disease }\end{array}$ & 6 & (1.7) & 12 & (3.5) & 109 & $(1.4)$ & $<0.01^{\mathrm{a}}$ \\
\hline & $\begin{array}{l}\text { Rheumatologic } \\
\text { disease }\end{array}$ & 12 & (3.6) & 22 & (6.4) & 222 & $(2.8)$ & $<0.01^{\mathrm{a}}$ \\
\hline & $\begin{array}{l}\text { Peptic ulcer } \\
\text { disease }\end{array}$ & 87 & (25.7) & 111 & $(32.3)$ & 1239 & (15.3) & $<0.01^{\mathrm{a}}$ \\
\hline & Liver disease & 49 & (14.5) & 80 & $(23.3)$ & 831 & (10.3) & $<0.01^{\mathrm{a}}$ \\
\hline & Diabetes & 83 & (24.6) & 117 & $(26.7)$ & 1269 & (15.5) & $<0.01^{a}$ \\
\hline & $\begin{array}{l}\text { Hemiplegia or } \\
\text { paraplegia }\end{array}$ & 3 & $(0.9)$ & 5 & (1.5) & 51 & $(0.6)$ & $0.17^{a}$ \\
\hline & Renal disease & 25 & (7.4) & 23 & (6.7) & 211 & (2.6) & $<0.01^{\mathrm{a}}$ \\
\hline & Solid tumor & 56 & (16.6) & 85 & $(24.7)$ & 862 & (10.5) & $<0.01^{\mathrm{a}}$ \\
\hline & Leukemia & 3 & $(0.9)$ & 1 & $(0.3)$ & 50 & $(0.6)$ & $0.61^{\mathrm{a}}$ \\
\hline & Lymphoma & 7 & (2.1) & 5 & (1.5) & 113 & (1.4) & $0.60^{\mathrm{a}}$ \\
\hline & Hypertension & 88 & $(26.0)$ & 123 & $(35.8)$ & 1460 & (18.1) & $<0.01^{a}$ \\
\hline
\end{tabular}




\begin{tabular}{|lllllllll|}
\hline & Asthma & 52 & $(15.4)$ & 58 & $(16.9)$ & 603 & $(7.5)$ & $<0.01^{\mathrm{a}}$ \\
\hline & Mental Disease & 32 & $(9.5)$ & 52 & $(15.1)$ & 650 & $(8.1)$ & $<0.01^{\mathrm{a}}$ \\
\hline
\end{tabular}

Note: $\mathrm{n}(\%)$ or mean (SD) Does not include missing data.
1. a) Chi-squared test
b) One-way ANOVA

ICD-10: I Certain infectious and parasitic diseases, II Neoplasms, III Diseases of the blood and blood-forming organs and certain disorders involving the immune mechanism, IV Endocrine, nutritional, and metabolic diseases, V Mental and behavioral disorders, VI Diseases of the nervous system, VII Diseases of the eye and adnexa, VIII Diseases of the ear and mastoid process, IX Diseases of the circulatory system, X Diseases of the respiratory system, XI Diseases of the digestive system, XII Diseases of the skin and subcutaneous tissue, XIII Diseases of the musculoskeletal system and connective tissue, XIV Diseases of the genitourinary system, XV Pregnancy, childbirth, and the puerperium, XVI Certain conditions originating in the perinatal period, XVII Congenital malformations, deformations, and chromosomal abnormalities, XVIII Symptoms, signs and abnormal clinical and laboratory findings not elsewhere classified, XIX Injury, poisoning, and certain other consequences of external causes, XXI Factors influencing health status and contact with health services

\section{The risk factors for 3-day ED revisit}

To examine the risk factor of group A, a multiple logistic regression analysis was conducted in those discharged from the ED after the initial visit (Table 3). The following factors were related to more frequent 3-day ED revisit after discharge from the same ED: having a history of same hospital use (odds ratio [OR] = 1.60, 95\% confidence interval $[\mathrm{Cl}]: 1.20-2.13)$, arriving at the ED by ambulance (OR $=1.62, \mathrm{Cl}: 1.23-2.12)$, having a common problem (e.g., fever, problems with medical devices, and consultations with referrals) $(\mathrm{OR}=2.02, \mathrm{Cl}: 1.45-2.81)$, and history of asthma (OR $=1.77, \mathrm{Cl}: 1.25-2.49)$.

Table 3 Multiple logistic regression for 3-day ED revisit among patients discharged from ED

\begin{tabular}{|c|c|c|c|c|}
\hline \multirow{4}{*}{$\begin{array}{l}\text { OR } \\
\text { Women }\end{array}$} & \multicolumn{4}{|c|}{ Patients discharged from ED } \\
\hline & \multicolumn{4}{|c|}{$\bigotimes n=8,754 \rrbracket$} \\
\hline & \multicolumn{2}{|c|}{$95 \% \mathrm{Cl}$} & \multirow{2}{*}{$\begin{array}{l}P \text {-value } \\
1.60)\end{array}$} & \multirow[b]{2}{*}{0.06} \\
\hline & 1.26 & $(0.99-$ & & \\
\hline Children & 1.01 & $(0.73-$ & 1.39) & 0.96 \\
\hline Adults (ref.) & - & - & & - \\
\hline Older adults & 0.74 & $(0.54-$ & 1.02) & 0.07 \\
\hline Using health care services & 1.31 & $(0.82-$ & 2.07) & 0.26 \\
\hline History of same hospital use & 1.60 & $(1.20-$ & $2.13)$ & $<0.01$ \\
\hline Presence of economic problem & 0.99 & $(0.39-$ & $2.55)$ & 0.99 \\
\hline Ambulance & 1.62 & $(1.23-$ & 2.12) & $<0.01$ \\
\hline Sign Common Problem & 2.02 & $(1.45-$ & 2.81) & $<0.01$ \\
\hline History of Congestive heart failure & 1.46 & $(1.00-$ & 2.13) & 0.05 \\
\hline History of Renal disease & 1.93 & $(1.16-$ & $3.20)$ & 0.01 \\
\hline History of Asthma & 1.77 & $(1.25-$ & 2.49) & $<0.01$ \\
\hline
\end{tabular}

Note: Adjusted by ED month visit and diagnosis of ED discharge.

\section{The risk factors for 30-day ED revisit}

To examine the risk factor of group B, a multiple logistic regression analysis was conducted for those who were discharged from the ED after the initial visit (Table 4). The following factors were related to more frequent 30-day ED revisit after discharge from the same ED: having a history of same hospital use $(\mathrm{OR}=2.88, \mathrm{Cl}: 2.05-4.04)$ and using health care services $(\mathrm{OR}=2.48, \mathrm{Cl}$ : 1.73-3.55). 
Table 4 Multiple logistic regression for 30-day ED revisit among patients discharged from ED

\begin{tabular}{|c|c|c|c|c|}
\hline \multirow{4}{*}{$\begin{array}{l}\text { OR } \\
\text { Women }\end{array}$} & \multicolumn{4}{|c|}{ Patients discharged from ED } \\
\hline & \multicolumn{4}{|c|}{$\nabla n=8,416 \rrbracket$} \\
\hline & \multicolumn{2}{|c|}{$95 \% \mathrm{Cl}$} & \multirow{2}{*}{$\begin{array}{l}\text { P-value } \\
1.24)\end{array}$} & \multirow[b]{2}{*}{0.83} \\
\hline & 0.97 & $(0.76-$ & & \\
\hline Children & 0.98 & $(0.68-$ & $1.42)$ & 0.93 \\
\hline Adults (ref.) & - & - & & - \\
\hline Older adults & 1.04 & $(0.77-$ & 1.40) & 0.81 \\
\hline Using health care services & 2.48 & $(1.73-$ & $3.55)$ & $<0.01$ \\
\hline History of same hospital use & 2.88 & $(2.05-$ & 4.04) & $<0.01$ \\
\hline Presence of economic problem & 1.96 & $(0.98-$ & $3.95)$ & 0.06 \\
\hline Ambulance & 1.00 & $(0.74-$ & $1.33)$ & 0.98 \\
\hline History of Asthma & 1.52 & $(1.09$ - & 2.12) & 0.01 \\
\hline History of solid tumor & 1.48 & $(1.07-$ & 2.04) & 0.02 \\
\hline History of Psychiatric disease & 1.52 & $(1.04-$ & $2.21)$ & 0.03 \\
\hline
\end{tabular}

Note: Excluding group A. Adjusted by ED visit month and diagnosis of ED discharge.

\section{Discussion}

This study investigated the rate of ED revisits its related factors in Japan for the general population among patients discharged from a university hospital ED. Among 8,754 patients who were discharged from February to December 2013. The 3-day ED revisit rate was 3.9\%. Risk factors of 3-day ED revisit were a history of same hospital use, arriving at the ED by ambulance, presenting a common problem (e.g., fever, problems with medical devices, and consultations with referrals), and history of asthma. And the 30-day ED revisit rate was 7.8\%. The characteristics of 30-day ED revisit group compared to no revisit group were having a history of same hospital use and using health care services.

\section{ED revisit rate}

The ED revisit rate in this study was $0.6 \%$ on the same day, $3.92 \%$ within three days, $7.8 \%$ within 30 days, and $11.7 \%$ within 90 days. In previous studies, ED revisit rate was 3.1\%-13.1\% within 3 days, $15.8 \%-22.4 \%$ within 30 days, $22 \%-34.0 \%$ within 90 days [4, 14]. Overall, the ED revisit rate in this study was lower than those commonly reported.

A previous study reported that the small size of the ED and the absence of a social worker were predictive factors for ED revisits [15]. In addition, according to a previous study, among patients who revisited the ED within three days after discharge, $32 \%$ visited a different ED from their initial visit [16].

The low rate of ED revisits might be affected by the fact that the hospital was a special function hospital, so some ED discharge patients might visit other hospitals, general outpatient clinics, or might have died. Additionally, since it was a university hospital, many specialists available at any given time might have collaborated between departments.

According to previous studies, the revisit rate within three days was considered to be an indicator of the quality of emergency medical care in other countries [4]. It is important to consider a 3-day ED revisit rate as a quality indicator of ED care in Japan, and it is necessary to explore the possibility that a 30-day ED revisit rate might be a quality indicator of collaboration of the ED with other community primary care providers.

\section{The risk factors for 30-day ED revisit after discharge}

This study identified risk factors for 3-day ED revisit, and the characteristics of the 30-day ED revisit group compared with no revisit group by using data at the time of initial ED visit. 
In the Comprehensive Care System in Japan, the number of patients with high medical needs at home is increasing [17]. For an aging society, transitional care is generally given to inpatients in Japan [17]. The current recommended steps of transitional care are: (1) screening assessment, (2) deciding on the appropriate level of support, and (3) service adjustment to inpatients upon hospitalization [18]. Thus, discharged patients may also be considered eligible for such transitional care, but there is no such exact system in Japan. Therefore, it is strongly recommended taking steps to provide such care for ED patients in order to identify the patient with the information at the time of initial ED visit to involve them in some intervention.

In what follows, we discuss the data based on Andersen's behavioral model [12].

\section{Predisposing Characteristics}

In this study, having a history of same hospital use was more frequently associated with 3-day ED revisit and 30-day ED revisit. As a background, the medical insurance system guarantees free access for patients to any domestic hospitals, specialists, and clinics in Japan [19]. According to a patient behavioral survey, patients select hospitals for reasons such as "introduction by a doctor," "good accessibility for transportation," and "offering highly specialized medical care" [20]. When patients have a history of same hospital use, they can easily choose to visit the same hospital's ED, even if the symptoms are unrelated to their chronic diseases. Therefore, even though patients are more inclined to visit larger hospitals, they should be encouraged to visit the primary emergency institutions of their local government first and be ensured that they will be referred to larger hospitals if required.

\section{Enabling Characteristics}

In this study, arriving at the ED by ambulance was associated with higher 3-day ED revisit. A previous study found that arriving at the ED by ambulance at the initial ED visit, can increase revisit [21]. One possible reason for this is that the experience of ED visit with the support of an ambulance lowered the psychological hurdle to using ED.

Using health care services (e.g., long-term insurance services and disability certificate) was also associated with higher 30-day ED revisit. A previous study reported that patients who need home care services tend to revisit the ED [22]. Activities of daily living (ADL) in these patients are often low, they are highly dependent on medical care and use long-term care insurances. A handicapped person should be put in the highrisk group for ED revisits. Moreover, these people have contact points with some home care providers, who can be trained to be more attentive and perform regular follow-ups for patients to discover the symptoms earlier. This seems very important for ensuring proper communication channels between the ED staff and home care providers.

\section{Need Characteristics}

In this study, presentation during the ED visit with common problems (e.g., fever, problems with medical devices, and consultations with referrals) was highly associated with 3-day ED revisit. In previous studies, fever was considered a risk factor for ED revisits [23]. According to a previous medical records survey in Japan, about half of the pediatric patients visited ED for fever, $20 \%$ of which had visited another pediatrician or another hospital's ED [24]. We observed that fever was a risk factor even after adjusting for age. As fever is a frequentlyoccurring symptom in the ED and many patients are discharged home with fever, it is necessary for ED staff to explain to patients and their families how to manage a fever at home and when to seek further medical advice.

Having a history of asthma was also significantly associated with more 3-day ED revisit. In a previous study, asthmatic attacks were considered to be predictors of ED revisit within 72 hours [25]. In this study, the patients not only had asthmatic attack at initial ED revisit, but also other diagnosis. Patients with a history of asthma in this study might have been part of the group of patients who would more frequently visit ED.

\section{Limitations}

There are two wider limitations to this study. First, this was a retrospective study of old data, implying a limit to the accuracy of data and that it might not be applicable to the current situation of these years. Second, this was a single-center study, thus, the result may not be generalized for nationwide or rural areas. Future studies should be designed to follow-up of the actual situation of ED revisits in community based, including other hospitals.

\section{Contribution to Emergency Nursing Practice}

The current state of scientific knowledge on patients' risk factors for 3-day/30-day ED revisit. The main findings of this research revealed that after ED discharge in Japan, factors related to a 3-day ED revisit were: history of same hospital use, arriving by ambulance, having a common problem, or history of asthma. The factors related to 30-day ED revisit were: using health care services and having a history of same hospital 
use. Key implications for emergency nursing practice from this research are related to the importance of taking care of patients with these factors during their initial ED visit in clinical and community settings to reduce the high rates of ED revisits.

\section{Further Study}

As revealed in this study, ED revisit patients had various predisposing, enabling, and need-based characteristics. Hence, in future studies, it would be necessary to discover a pattern for reasons for ED revisit among patients, clarifying associated factors with each ED revisit reason. ED revisit rate should be further assessed by analyzing medical claim data to link them to individuals and clarify patient medical/homecare services use behavior.

\section{Conclusion}

This study identified the first statistical evaluation of ED revisit after discharge from the same ED in Japan which was not specific to a certain class of disease or age. Among the patients who were discharged the ED from February to December $2013,3.9 \%$ patients revisited the same ED within three days, and 7.8\% patients revisited within 30 days. The factors related to 3-day ED revisit were history of same hospital use, arriving at the ED by ambulance, presenting with common problems, and a history of asthma. The factors related to 30-day ED revisit were history of same hospital use and using health care services. Attention should be paid to patients with these factors during their initial ED visit.

\section{Abbreviations}

ED: Emergency Department

OECD: Organization for Economic Cooperation and Development

RECORD: Reporting of studies Conducted using Observational Routinely-collected health Data

JTAS: Japan Triage and Acuity Scale

\section{Declarations}

\section{Ethics approval and consent to participate}

This study was approved by the ethics review committee of the Faculty of Medicine of The University of Tokyo (approval number: 10593 , July 2014). The consent to participate was obtained in the form of opt-out on the web-site. Those who rejected were excluded.

\section{Consent for publication}

Not applicable

\section{Availability of data and materials}

Not applicable

\section{Competing Interests}

The authors declare that they have none.

\section{Funding}

This work was supported by the research grant program of The Univers Foundation, The Univers Foundation, Shinjuku-ku, Tokyo Grant Number 15-01-143 and JSPS KAKENHI Grant Numbers JP17H06650, JP19K19731.

\section{Authors' contributions}

CT, SN, and SY collected the medical chart information of ED patients, CT analyzed, CT, SN, TN, SN, SY, MG, TS, SI, and NYM interpreted the patient data regarding ED visit. CT, SN, TN, and NYM were a major contributor in writing the manuscript. All authors read and approved the final manuscript.

\section{Acknowledgements}


We appreciate the ED staff of The University of Tokyo Hospital for their advice and cooperation. We are also sincerely grateful to the biostatistics lecturer at the Department of Public Health and Medical Sciences, Graduate School of Medicine, The University of Tokyo, for their advice on the analyses.

\section{References}

1 Berchet C. Emergency care services: trends, drivers and interventions to manage the demand. OECD Health Working Papers No. 83 2015:149. doi:10.1787/5jrts344crns-en

2 Ministry of Health, Labor and Welfare. https://www.mhlw.go.jp/content/10802000/000328610.pdf. Accessed 30 March 2020 [in Japanese].

3 D'Avolio DA, Feldman J, Mitchell P, et al. Access to care and health-related quality of life among older adults with nonurgent emergency department visits. Geriatr Nurs. 2008;29:240-6. doi: 10.1016/j.gerinurse.2008.06.005

4 Han CY, Chen LC, Barnard A, et al. Early Revisit to the Emergency Department: An Integrative Review. J Emerg Nurs. 2015;41:285-95. doi: 10.1016/j.jen.2014.11.013

5 McCusker J, Bellavance F, Cardin S, et al. Detection of older people at increased risk of adverse health outcomes after an emergency visit: the ISAR screening tool. J Am Geriatr Soc. 1999;47:1229-37. doi: 10.1111/j.1532-5415.1999.tb05204.x

6 Nakamura M, Ihara N, Ogino T, et al. Actual of suicide attempt patients housed in Gunma University Medical School affiliated hospital. Kita Kanto Igaku 2006;56:113-7. [in Japanese]

7 Matsumoto T, Fujita J, Nakamura H, et al. ER Project. Evaluation of patient education at the time of ER visit, usefulness of introduction of inhaled corticosteroid / compound. Okinawa Med J. 2010;48:35-8 [in Japanese].

8 Ministry of Health, Labor and Welfare. About review of discharged patient survey. http://www.mhlw.go.jp/file/05-Shingikai-12404000Hokenkyoku-Iryouka/0000096122.pdf. Accessed 30 March 2020 [in Japanese].

9 Naylor M, Keating SA. Transitional Care: Moving patients from one care setting to another. Am J Nurs. 2009;108:58-63.

10 Benchimol El, Smeeth L, Guttmann A, Harron K, Moher D, Petersen I, Sørensen HT, von Elm E, Langan SM; RECORD Working Committee. The Reporting of studies Conducted using Observational Routinely-collected health Data (RECORD) Statement. PLoS Med. 2015;12(10):e1001885. doi: 10.1371/journal.pmed.1001885.

11 The University of Tokyo hospital. Number of patients. https://www.h.u-tokyo.ac.jp/about/kanjasu/. Accessed 30 March 2020 [in Japanese].

12 Andersen R, Davidson P. Improving Access to Care in America: Individual and Contextual Indicators. In: Andersen R, Rice T, Kominski G Eds. Changing the U.S. health care system: Key issues in health services policy and management. San Francisco, CA: Jossey-Bass 2007:3-31.

13 Japanese Association for Acute Medicine, Japanese Association for Emergency Nursing, Japanese Society for Emergency Medicine. Japan Triage and Acuity Scale 2012 Guidebook. Tokyo: Herusu shuppan; 2016 [in Japanese].

14 Moons P, De Ridder K, Geyskens K, et al. Screening for risk of readmission of patients aged 65 years and above after discharge from the emergency department: predictive value of four instruments. Eur J Emerg Med. 2007;14:315-23. doi: 10.1097/MEJ.0b013e3282aa3e45

15 McCusker J, lonescu-Ittu R, Ciampi A, et al. Hospital characteristics and emergency department care of older patients are associated with return visits. Acad Emerg Med. 2007;14:426-33.

16 Duseja R, Bardach NS, Lin GA, et al. Revisit rates and associated costs after an emergency department encounter: a multistate analysis. Ann Intern Med. 2015;162:750-6. doi: 10.1197/j.aem.2006.11.020

17 Ministry of Health, Labor and Welfare. Basic information on community health care in 2009.

http://www.mhlw.go.jp/toukei/saikin/hw/hoken/kiso/21.html. Accessed 30 March 2020 [in Japanese].

18 Utsunomiya H. Discharge support practical navigation. Tokyo: IGAKU-SHOIN; 2015. 18-19. [in Japanese]

19 Ministry of Health, Labor and Welfare. About medical insurance in our country. 2016;

http://www.mhlw.go.jp/stf/seisakunitsuite/bunya/kenkou_iryou/iryouhoken/iryouhoken01/. Accessed 20 Nov 2019 [in Japanese].

Page $12 / 14$ 
21 Friedmann PD, Jin L, Karrison TG, Hayley DC, Mulliken R, Walter J, et al. Early revisit, hospitalization, or death among older persons discharged from the ED. American Journal of Emergency Medicine. 2001;19(2):125-9.

22 McCusker J, Cardin S, Bellavance F, et al. Return to the emergency department among elders: patterns and predictors. Acad Emerg Med. 2000;7:249-59. doi:10.1111/j.1553-2712.2000.tb01070.x

23 Wu CL, Wang FT, Chiang YC, et al. Unplanned emergency department revisits within 72 hours to a secondary teaching referral hospital in Taiwan. J Emerg Med. 2010;38:512-7. doi: 10.1016/j.jemermed.2008.03.039

24 Taga T, TanakaY, Hashimoto K, et al. Consideration of a case of visiting our hospital emergency outpatient (pediatric department) with chief complaint of fever. Comparison between our case where we visited our department of pediatrics during the day and the case where we visited another hospital during the day. Japanese J Pediatr. 2001;54:1497-501 [in Japanese].

25 Cyounabayashi N, Sugiyama K, Kato M, et al. The actual condition of bronchial asthma patients in emergency outpatient and the influence of inhaled corticosteroid prescription. Multicenter cohort observation study. Japanese J Allergol. 2010;59:123-36 [in Japanese].

\section{Figures}

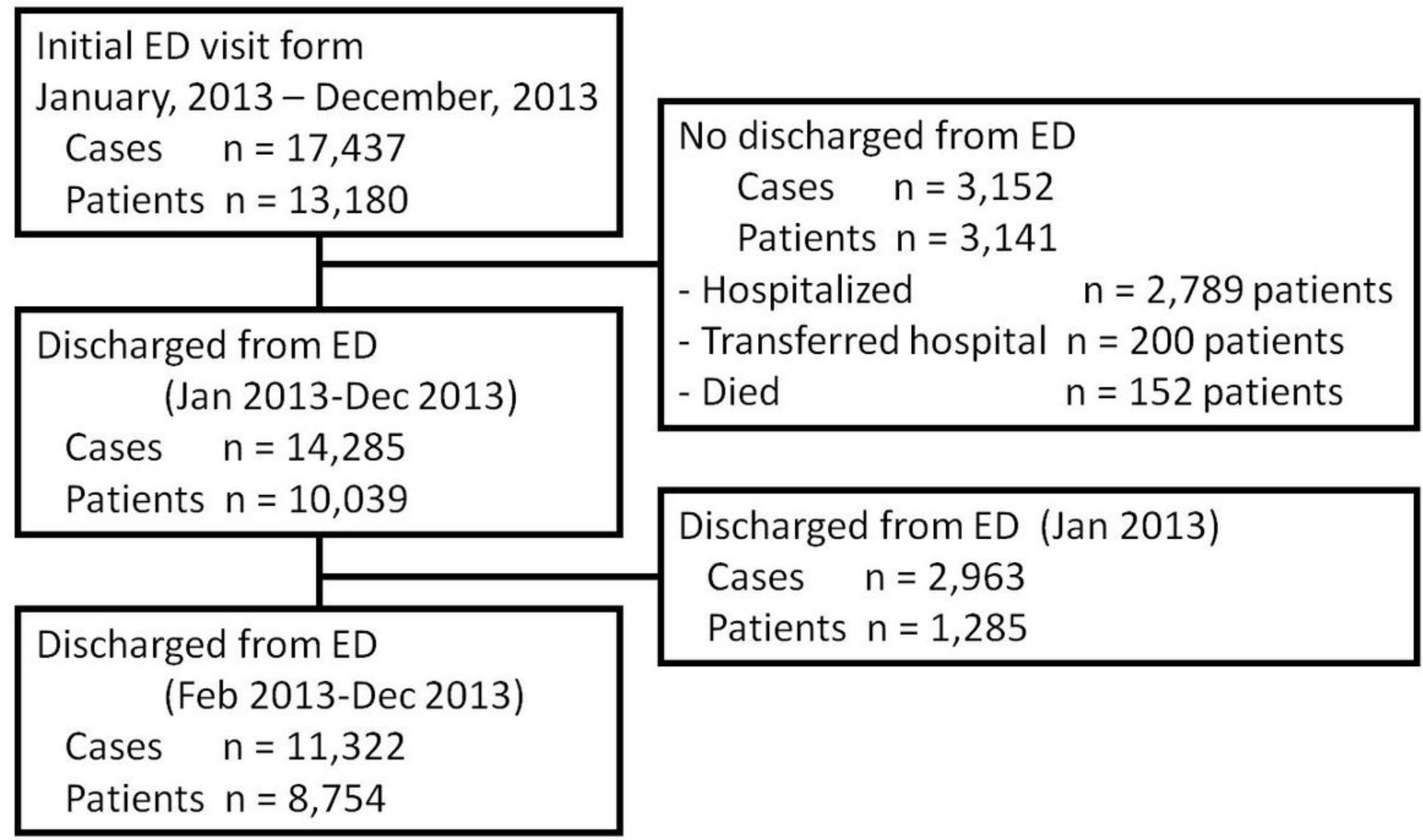

Figure 1

Flow of participants 


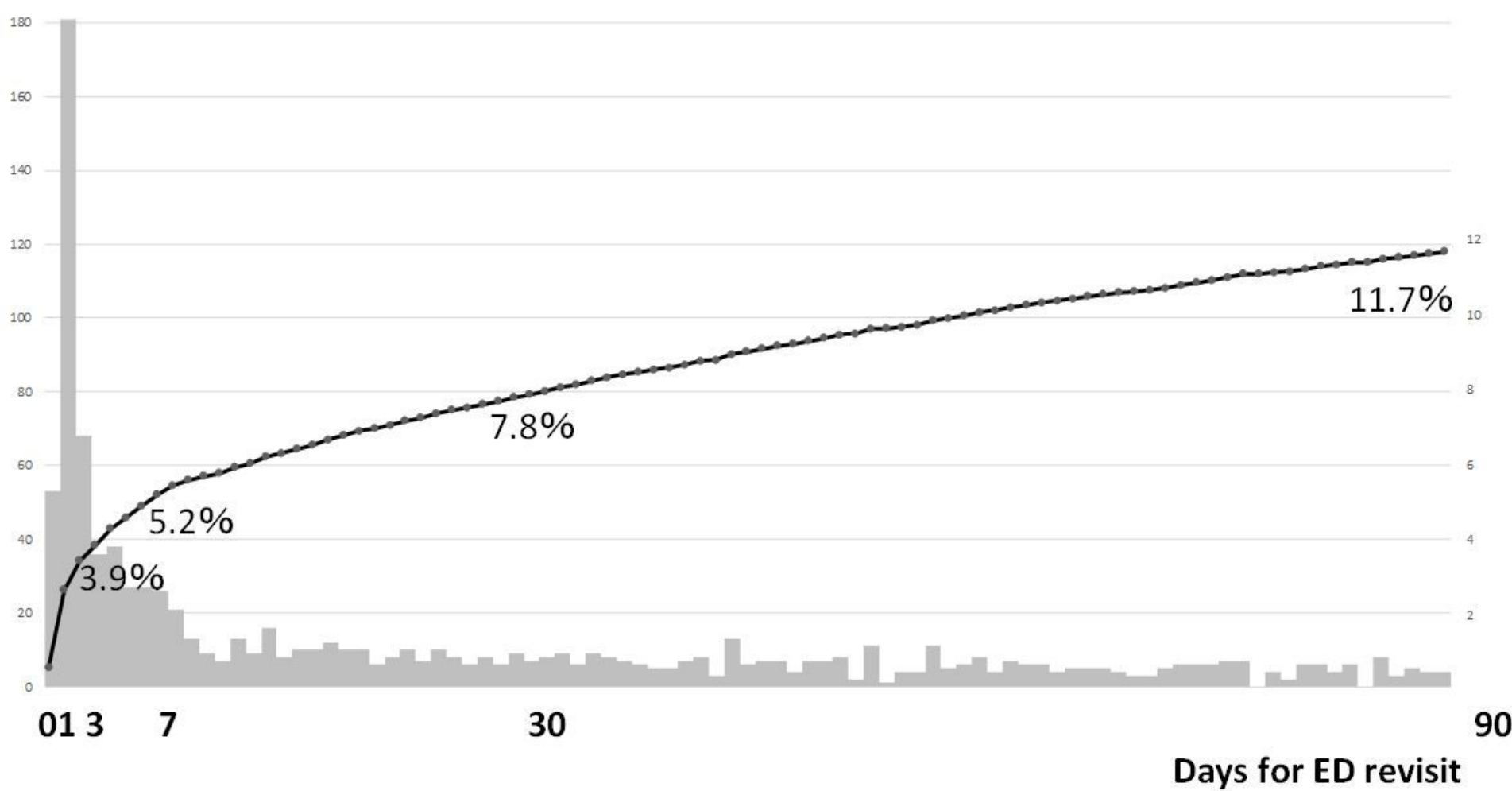

\section{Figure 2}

Transition of emergency department revisits within 90 days after discharge

\section{Supplementary Files}

This is a list of supplementary files associated with this preprint. Click to download.

- Additionalfile1.docx 